\title{
Caracterización elemental del alquitrán del cigarro por medio de espectroscopia de fluorescencia de rayos- $X$ dispersiva en energía
}

\section{Elementary characterization of tar of cigar by means of energy dispersive X-ray fluorescence spectroscopy}

\author{
Paolo G. Tataje, Daniel A. Muñoz, Jorge A. Bravo
}

Facultad de Ciencias Físicas, Universidad Nacional Mayor de San Marcos, Av. Venezuela S/N, Lima, Perú.

DOI: https://doi.org/10.33017/RevECIPeru2016.0001/

\section{Resumen}

Se analizó una muestra de alquitrán por medio de la técnica de fluorescencia de rayos-X dispersiva en energía (FRXDE) con la finalidad de obtener en forma cuantitativa la composición elemental inorgánica que este presenta. La sustancia tóxica se extrajo de una marca de cigarro genérica mediante un experimento que simula, de forma muy eficiente, los pulmones de un fumador. Los resultados del presente trabajo enmarcan una diferencia entre el tabaco y lo que se obtiene de la combustión de este ingresando a los pulmones

Descriptores: alquitrán, fluorescencia de rayos-x dispersiva en energía, composición elemental.

\section{Abstract}

A Tar sample was analyzed by energy dispersive X-ray fluorescence spectroscopy (EDXRF) in order to obtain quantitatively its elemental inorganic composition the elemental composition. The toxic substance was extracted from a generic brand of cigar through an experiment that simulates, very efficiently, the lungs of a smoker. The results of this work show a difference between tobacco and what is obtained from the combustion entering the lungs.

Keywords: tar, energy dispersive X-ray fluorescence spectroscopy, elementary composition.

\section{Introducción}

Debido al incremento del consumo de tabaco en el mundo se han desarrollado múltiples estudios sobre la composición elemental que este presenta, entre estos estudios se han identificado la presencia de metales e incluso de radioisótopos [1]. Nuestro país no es ajeno al incremento de consumo de esta sustancia nociva, motivo por el cual se decidió realizar el siguiente trabajo con la finalidad de enmarcar las diferencias entre la composición del tabaco y la del humo que los fumadores activos llevan a sus pulmones.
Es necesario indicar que la composición de las hojas de tabaco depende del lugar de donde han sido extraídas [2], debido a ello es que no todos los cigarros están compuestos por la misma concentración de elementos y por consiguiente el humo que ingresa a los pulmones también varía con respecto a cada cigarro. Sin embargo, lo que pretende el presente trabajo es caracterizar de forma cualitativa y cuantitativa la composición elemental inorgánica de esta sustancia.

En la actualidad la Facultad de Ciencias Físicas cuenta con un equipo portátil de fluorescencia de rayos- $X$ dispersiva en energía, adquirido de la empresa AMPTEK, cuya fuente de radiación cuenta 
con un ánodo de oro. La cadena de detección de rayos- $X$ de baja energía del equipo tiene una resolución de 140 eV a media altura para rayos- $X$ de $6.40 \mathrm{keV}$. El detector utiliza un cristal de Si-PIN de aproximadamente $500 \mu \mathrm{m}$ de espesor y $3 \mathrm{~mm}$ de diámetro. Este cristal da lugar a un peculiar fenómeno llamada "pico de escape" que será tomado muy en cuenta para evitar la confusión entre estos picos y los rayos-X característicos [3].

\section{Sedimentación, disolución y obtención del alquitrán}

\subsection{Sedimentación y disolución en agua}

En esta parte del experimento se utilizaron 100 cigarrillos de una marca genérica de los cuales se comenzaría a extraer el alquitrán de manera análoga a un fumador activo. Para ello se montó una pequeña máquina que simule estas condiciones, esta máquina mediante una fuente de absorción de aire conectada, de forma hermética, entre dos matraces era capaz de fumar el cigarro, que se encontraba en la boca de uno de los matraces. Ambos contenían agua a media altura, parte del humo de cigarro que pasaba por el agua se precipitaba mientras que otra porción se comenzaba a disolver en él.

\subsection{Obtención del alquitrán}

Luego de haber realizado la primera parte del experimento, se pasó a destapar los matraces, que contenían el agua turbia producto de la disolución con alquitrán, y mezclar su contenido en un vaso de precipitado.

Se calentó la muestra hasta aproximadamente 100 -C evaporando el agua y dejando de residuo el alquitrán. La muestra obtenida se colocó en una luna de reloj y se sometió en un horno a $100^{\circ} \mathrm{C}$, con la finalidad de evaporar el resto de agua presente.

\section{Aplicación de técnica nuclear}

Se irradió la muestra con un continuo de energías que van desde los $0-25 \mathrm{keV}$ durante aproximadamente 300 segundos, obteniendo para elementos livianos rayos- $X$ característicos $\mathrm{K} \alpha$ y $\mathrm{K} \beta$, mientras que para elementos pesados obtuvimos rayos- $X$ característicos $L$ y $M$.

Posterior a ello se colocó la muestra a pocos milímetros del detector, estando esta de forma perpendicular a la ventana del detector, para observar picos debido a elementos radiactivos presentes en esta.

\subsection{Caracterización cualitativa y cuantitativa}

El análisis cualitativo del espectro se realiza por medio de las energías de los picos obtenidos. Este análisis es óptimo y un pre-paso para realizar la cuantificación, debido a que la concentración en masa de los elementos es proporcional a la intensidad de los picos de rayos-X característicos.

Tabla 1: Energía de los rayos- $X$ característicos obtenidos en la muestra.

\begin{tabular}{cccc}
\hline $\begin{array}{c}\text { Símbolo } \\
\text { del } \\
\text { elemento }\end{array}$ & $\begin{array}{c}\text { Energía } \\
\mathrm{Ka} \\
(\mathrm{keV})\end{array}$ & $\begin{array}{c}\text { Energía } \\
\mathrm{K} \beta \\
(\mathrm{keV})\end{array}$ & $\begin{array}{c}\text { Energía } \\
\mathrm{La} \\
(\mathrm{keV})\end{array}$ \\
\hline $\mathrm{Si}$ & 1.739 & 1.829 & - \\
$\mathrm{S}$ & 2.307 & 2.464 & - \\
$\mathrm{Cl}$ & 2.621 & 2.815 & - \\
$\mathrm{Ar}$ & 2.957 & 3.190 & - \\
$\mathrm{K}$ & 3.312 & 3.589 & - \\
$\mathrm{Ca}$ & 3.690 & 4.012 & 0.341 \\
$\mathrm{Mn}$ & 5.894 & 6.489 & 0.637 \\
$\mathrm{Fe}$ & 6.398 & 7.057 & 0.705 \\
$\mathrm{Cu}$ & 8.040 & 8.905 & 0.923 \\
$\mathrm{Zn}$ & 8.630 & 9.572 & 1.012 \\
$\mathrm{Sr}$ & 14.140 & 15.836 & 1.807 \\
$\mathrm{Zr}$ & 15.744 & 17.668 & 2.042 \\
$\mathrm{Ba}$ & 32.062 & 36.378 & 4.466 \\
\hline
\end{tabular}

La Tabla 1 muestra los valores de energía de los rayos-X característicos [4] obtenidos en el espectro, cabe indicar que debido a que el potencial nominal es de aproximadamente $25 \mathrm{kV}$ no se podrán apreciar los picos $\mathrm{Ka}$ y $\mathrm{K} \beta$ de $\mathrm{Ba}$ cuyo potencial de ionización de la capa-K es mayor que $25 \mathrm{keV}$.

La caracterización cuantitativa se realizó por medio de un programa de simulación llamado SimXcom2015 que calcula con respecto a la concentración de los elementos, la intensidad que deberían de tener los picos y la producción de los "picos de escape" [3]. Este programa, que pertenece al Laboratorio de Arqueometría de la Facultad de Ciencias Físicas, tiene datos sobre las propiedades atómicas y las secciones eficaces [3] que se han obtenido accediendo a los portales web de la OIEA [5] y del NIST [6]. 


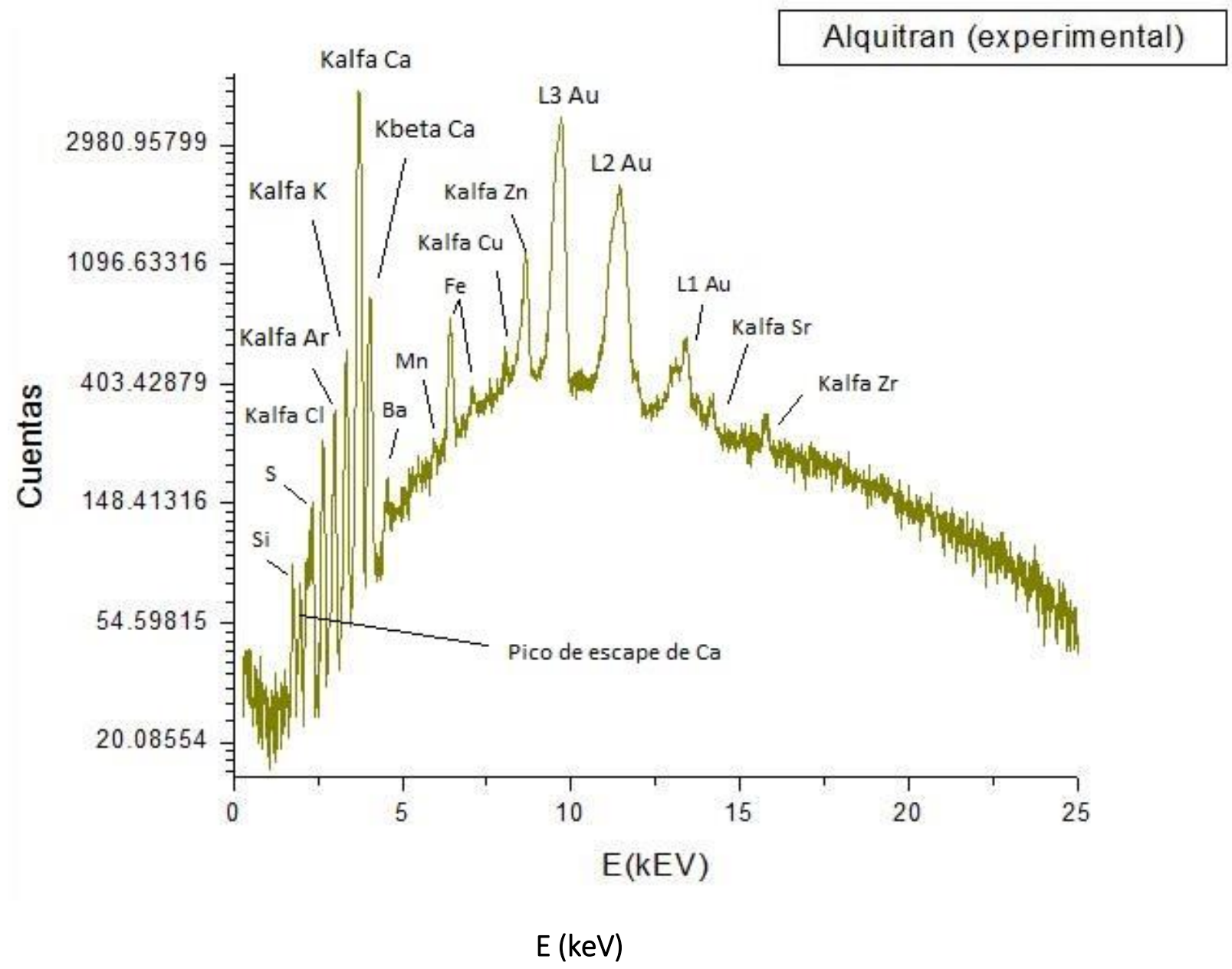

Figura 1: Espectro del alquitrán del cigarro irradiado con una fuente de rayos- $X$ con ánodo de oro.

Tabla 2: Concentración en masa de los elementos presentes en la muestra.

\begin{tabular}{cccc}
\hline $\begin{array}{c}\text { Símbolo del } \\
\text { elemento }\end{array}$ & $\begin{array}{c}\text { Total de rayos- } X \\
\text { simulados }\end{array}$ & $\begin{array}{c}\text { Concentración } \\
\text { en masa }(\%)\end{array}$ & $\begin{array}{c}\text { Incertidumbre } \\
(\%)\end{array}$ \\
\hline $\mathrm{Si}$ & 526 & 1.8095 & 0.0829 \\
$\mathrm{~S}$ & 1158 & 0.5160 & 0.0168 \\
$\mathrm{Cl}$ & 2125 & 0.4130 & 0.0107 \\
$\mathrm{Ar}$ & 1030 & 0.1585 & 0.0054 \\
$\mathrm{~K}$ & 4933 & 0.2609 & 0.0052 \\
$\mathrm{Ca}$ & 50409 & 1.6180 & 0.0240 \\
$\mathrm{Mn}$ & 1097 & 0.0075 & 0.0003 \\
$\mathrm{Fe}$ & 6128 & 0.0285 & 0.0005 \\
$\mathrm{Cu}$ & 3476 & 0.0069 & 0.0002 \\
$\mathrm{Zn}$ & 13167 & 0.0285 & 0.0005 \\
$\mathrm{Sr}$ & 628 & 0.0029 & 0.0001 \\
$\mathrm{Zr}$ & 914 & 0.0056 & 0.0002 \\
$\mathrm{Ba}$ & 1711 & 0.0380 & 0.0011 \\
\hline
\end{tabular}




\section{Resultados y discusión}

Se determinó con muy baja incertidumbre la concentración en masa de los elementos presentes en la muestra de alquitrán, considerando que los elementos orgánicos ocupan más del $90 \%$ de la concentración, estos resultados se enuncian en la Tabla 2.

No se encontró rastro de radioisótopos, sin embargo, eso no indica completamente la ausencia de estos debido a que puede haber un emisor beta [7] o alfa y este no ser identificado por la técnica utilizada.

Los elementos más abundantes en la muestra son $\mathrm{Ca}$ [8] y Si. Sin embargo, notamos que el pico del $\mathrm{K} \alpha$ de Si es de muy poca intensidad, esto se debe a que el proceso de emisión de un rayo- $X$ característico compite con otro proceso llamado efecto Auger, el cual es no radiactivo y consiste en un reacomodo de sus electrones emitiendo uno de ellos (electrón Auger), para elementos livianos predomina este efecto. En el Si la probabilidad que se de emisión de rayos- $X$ característicos es del $5 \%$ [5].

En la Figura 1 notamos la presencia de picos de $\mathrm{Au}$ que corresponden al ánodo de la fuente $\mathrm{y}$ también la presencia del "pico de escape" de Ca, que corresponde a la energía de $1.95 \mathrm{keV}$. Este pico puede ser confundido con el pico del Ka de $\mathrm{P}$ que se encuentra muy cercano en energía, siendo esta $2.013 \mathrm{keV}$.

El pico del Ka de Mn se encuentra pronunciado, la abundancia de este elemento con referente a otros trabajos [9], radica en el fundamento de que las hojas de tabaco varían su composición según el lugar de donde han sido extraídas. [2]

\section{Conclusiones}

Los resultados cualitativos obtenidos de las concentraciones son muy similares a otros trabajos análogos, sin embargo la diferencia radica en los resultados cuantitativos, puesto a que en el presente trabajo se analizó el humo, producto de la combustión en el tabaco, que ingresa a los pulmones. Es por ello que a partir de esto se concluyó que la concentración de elementos que ingresan a los pulmones es menor que la concentración de elementos que se encuentra en el propio cigarro, parte de esta concentración puede haber sido depositada en los conductos respiratorios.

\section{Agradecimientos}

Se agradece al Dr. Jorge Bravo por la ayuda prestada y al Laboratorio de Arqueometría por haber hecho posible este trabajo al permitirnos usar el espectrómetro de fluorescencia de rayos- $X$ dispersiva en energía.

\section{Referencias}

[1] Mendoza, M. y Olivera, P. (2013). "Análisis comparativo de elementos tóxicos en tabaco mediante técnicas analíticas de fluorescencia de rayos X" en Informe Científico Tecnológico, vol 13, ISSN 16841662, p. 31-35.

[2] Rehman, A., Nasiruddin, M., Sarwar, A. and Bhutto, S. (2014). "Physicochemical Analysis of Different Cigarettes Brands Available in Pakistan" in Pakistan Journal of Analytical \& Environmental Chemistry, vol. 15, No. 2, ISSN-1996-918X, p. 26-38.

[3] Bravo, J. (2015). "Dependencia en energía de la producción de picos de escape en espectroscopia de fluorescencia de rayos- $X$ dispersiva en energía" en Revista de Investigación de Física, vol. 18, 151801252, p. 1-5.

[4] Thompson, A., Attwood, D., Gullikson, E., Howells, M., Kim, K., Kirz, J., Kortright, J., Winick, H., Lindau, I., Pianetta, P., Robinson, A., Scofield, J., Underwood, J., Vaugham, D. and Williams, G. (2001). XRAY DATA BOOKLET. California: Lawrence Berkeley National Laboratory.

[5] OIEA, Quantitative X-ray Analysis System, Agencia Internacional de Energía Atómica, Viena (1995).

[6] M. J. Berger, XCOM: Photon Cross Sections Database, National Institute of Standard and Techn ology, Washington (1998).

[7] Jenkins, R., Newman, R., Lester, G., Frish, A. and Williamson, T. (1982). "Neutron Activation Analysis in Tobacco and Cigarette Smoke Studies- The Halogens" in Beiträge zur Tabakforschung International, vol. 11, No. 4, Issue 4, p. 195-202.

[8] Martinez, T., Lartigue, J., Zarazua, G., Avila-Perez, P., Navarrete, M. and Tejeda, S. (2008). "Application of the Total Reflection X-ray Fluorescence Technique to 
trace elements determination in tobacco" in Spectrochimica Acta Part B, vol. 63, p. 1469-1472.

[9] Lartigue, J., Martinez, T., Avila-Perez, P., Zarazua, G. and Tejeda, S. (2007). "EDXRF analysis of some samples of cigarette paper" in Journal of Radioanalytical and
Nuclear Chemistry, vol. 272, No. 3, p. 759762.

E-mails: ptataje@yahoo.com,

amunozgambini@gmail.com, ibravo8@hotmail.com 